\title{
Neuromyelitis Optica Spectrum Disorder: Clinical Burden and Cost of Relapses and Disease-Related Care in US Clinical Practice
}

\author{
Minying Royston · Adrian Kielhorn • Derek Weycker • \\ Melody Shaff · Linnea Houde · Imran Tanvir · Shamik Bhattacharyya • \\ Michael Levy
}

Received: March 2, 2021 / Accepted: April 22, 2021 / Published online: May 27, 2021

(C) The Author(s) 2021

\section{ABSTRACT}

Introduction: Neuromyelitis optica spectrum disorder (NMOSD) is a rare autoimmune condition characterized by unpredictable relapses that affect the optic nerves and spinal cord, which can lead to blindness, paralysis, and increased mortality rates. Evidence on the clinical and economic burden of NMOSD in the USA is currently lacking.

Methods: A retrospective, observational cohort study was conducted using data from the IQVIA PharMetrics Plus Healthcare Claims Database between January 1, 2012 and March 31, 2019.

Supplementary Information The online version contains supplementary material available at https:// doi.org/10.1007/s40120-021-00253-4.

M. Royston · A. Kielhorn · I. Tanvir Alexion Pharmaceuticals Inc., Boston, MA, USA

D. Weycker $(\varangle) \cdot$ M. Shaff · L. Houde Policy Analysis Inc. (PAI), 822 Boylston Street, Suite 206, Chestnut Hill, MA 02467, USA

e-mail: dweycker@pai2.com

S. Bhattacharyya

Brigham and Women's Hospital, Boston, MA, USA

S. Bhattacharyya $\cdot$ M. Levy

Harvard Medical School, Boston, MA, USA

M. Levy

Massachusetts General Hospital, Boston, MA, USA
Adults (aged 18 years or more) with evidence of NMOSD and a matched group of comparison patients were identified. Outcomes, including NMOSD relapses, healthcare utilization, and healthcare expenditure (reported in 2018 US dollars), were evaluated during the follow-up period (maximum 6 years). Healthcare utilization and expenditure were assessed overall (allcause) and during NMOSD relapses.

Results: The study included 1363 patients with NMOSD; the mean age was 44.9 years, and $75.3 \%$ were female. During the follow-up period (median 2.0 years), $47.7 \%$ of patients with NMOSD had one or more relapses, corresponding to an annualized relapse rate of 0.8 (95\% confidence interval [CI] 0.7-0.9). When analyzing healthcare expenditure per patient, the mean annualized all-cause healthcare expenditure among patients with NMOSD was $\$ 60,599$ (95\% CI \$52,112-66,716) compared with $\$ 8912$ (95\% CI \$7084-10,727) among comparison patients, representing a difference of $\$ 51,687$ $(95 \%$ CI $\$ 43,820-58,664)$ attributable to NMOSD. The mean annualized total expenditure for NMOSD relapses was \$10,070 (95\% CI \$7726-12,660) per patient, with hospital/inpatient care requiring more expenditure than ambulatory/outpatient care.

Conclusion: Findings of this large, retrospective, observational study indicate that relapses among patients with NMOSD are common in US clinical practice, leading to substantial healthcare utilization and expenditure. 
Therapies with the highest relapse risk reduction could lead to markedly lower relapse-associated healthcare utilization and clinical burden in patients with NMOSD.

\section{PLAIN LANGUAGE SUMMARY}

Neuromyelitis optica spectrum disorder (NMOSD) is a severely debilitating neurological disease that affects the nerves in the brain and spinal cord. People who have NMOSD may experience recurrent attacks, or relapses, that can cause blindness and disability. These relapses may lead to hospitalizations, doctor's office visits, and pharmacy costs that are paid by health insurance plans. Overall, the cost of treating relapses in patients with NMOSD is substantial. Our study analyzed healthcare claims data from the USA. During a median follow-up time of 2.0 years, our study showed that $47.7 \%$ of patients with NMOSD experienced one or more relapses, resulting in hospital/inpatient admissions and ambulatory/ outpatient treatments. In addition, the average healthcare cost among patients with NMOSD was $\$ 60,599$ per year compared with $\$ 8912$ per year for patients without NMOSD. This represents a difference of $\$ 51,687$ per year, which can be attributed to NMOSD. Among patients with three or more relapses during the followup period, the average total healthcare cost was more than $\$ 83,000$ per patient. Therefore, medicines that prevent relapses could lead to fewer relapse-associated hospitalizations and outpatient treatments for patients with NMOSD.

Keywords: Cost of illness; Disease burden; Economic burden; Healthcare costs; Healthcare resource utilization; Neuromyelitis optica; Neuromyelitis optica spectrum disorder; Optic neuritis; Relapses; Transverse myelitis

\section{Key Summary Points}

\section{Why carry out this study?}

Despite advancements in the diagnosis and treatment of neuromyelitis optica spectrum disorder (NMOSD), much remains unknown concerning the clinical and economic burdens of NMOSD relapses, which have been shown to drive disease progression.

Using data from a large healthcare claims database in the USA, a retrospective, observational cohort study was conducted to assess healthcare utilization and healthcare expenditure in patients with NMOSD-both overall (all-cause) and during NMOSD relapses.

\section{What was learned from the study?}

The mean annualized all-cause healthcare expenditure among patients with NMOSD was $\$ 60,599$ (95\% CI \$52,112-66,716) compared with $\$ 8912$ (95\% CI \$7084-10,727) among comparison patients, representing a mean difference of $\$ 51,687$ (95\% CI $\$ 43,820-58,664)$ attributable to NMOSD. Of patients with NMOSD, 47.7\% had one or more relapses, with those who had three or more relapses accruing mean annualized healthcare expenditures of over $\$ 83,000$.

Therapies with the highest relapse risk reduction could lead to markedly lower relapse-associated healthcare utilization and clinical burden in patients with NMOSD.

\section{DIGITAL FEATURES}

This article is published with digital features, including a summary slide and plain language summary, to facilitate understanding of the 
article. To view digital features for this article go to https://doi.org/10.6084/m9.figshare. 14459586.

\section{INTRODUCTION}

Neuromyelitis optica spectrum disorder (NMOSD) is a rare, chronic, autoimmune condition of the central nervous system (CNS) that causes demyelination and axonal damage to the optic nerves and spinal cord $[1,2]$. The disorder usually causes optic neuritis or transverse myelitis and is strongly associated with the presence of anti-aquaporin-4 (AQP4) antibodies. According to the International Panel for Neuromyelitis Optica (NMO) Diagnosis, NMOSD is associated with serological antibodies to AQP4 (AQP4+) in more than $75 \%$ of cases, and is $99 \%$ specific for making the diagnosis in the context of one or more core clinical characteristics including optic neuritis, transverse myelitis, area postrema syndrome, acute brainstem syndrome, narcolepsy, and cerebral syndrome [2]. In patients without AQP4+ antibody status, evidence of lesions by magnetic resonance imaging and two or more core clinical characteristics are required for diagnosis [2]. Thus, core clinical characteristics are essential to help differentiate NMOSD from multiple sclerosis (MS) and other CNS disorders.

Patients with NMOSD experience recurrent, unpredictable, and debilitating relapses that can lead to blindness, paralysis, and increased mortality. Acute relapses are typically treated intravenously with methylprednisolone for 3-5 days, with complete recovery in only $17-35 \%$ of cases; intravenously administered immunoglobulin and plasma exchange are useful second-line treatments, which may be used to improve outcomes [1, 3-6]. To prevent relapses, immunosuppressive agents, such as azathioprine, mycophenolate mofetil, and rituximab, have historically been used off-label in clinical practice. Several newer agents (eculizumab, inebilizumab, and satralizumab) have recently been approved for the treatment of NMOSD in AQP4+ patients [1, 2, 7-10].

Despite advancements in the diagnosis and treatment of NMOSD, much is yet unknown concerning the clinical and economic burden of disease. Challenges to ascertaining AQP4 serostatus and multiple revisions to the diagnostic criteria for NMOSD may have contributed to disparate estimates of disease burden across the little evidence that is available [11]. In addition, although studies have evaluated healthcare utilization on an overall basis, there are few studies investigating the burden of disease based on the frequency and severity of NMOSD relapses in large patient cohorts [12-14]. Therefore, studies are needed to understand the real-world impact of NMOSD relapses in clinical practice. Using data from a large US healthcare claims database, a retrospective, observational cohort study was conducted to assess healthcare utilization and expenditure in patients with NMOSD, both overall (all-cause) and during NMOSD relapses.

\section{METHODS}

\section{Study Design and Data Source}

A retrospective, observational, cohort study was conducted using data from the IQVIA PharMetrics Plus Healthcare Claims Database between January 1, 2012 and March 31, 2019. The database contains medical and pharmacy claims from over 75 private health plans, covering more than 40 million people annually across the USA. The database includes patientlevel data on dates and places of medical service, diagnosis and procedure codes, and pharmacy data such as drug codes, dispensation dates, dosages, and drug supply. Health plan expenditure for services rendered and selected demographic information (age, sex, geographic region of residence, dates of plan eligibility) are also available. Patient-level data can be arrayed longitudinally to provide a detailed profile of all medical and pharmacy services used by each patient over time.

The study database was de-identified before its release to study investigators, as set forth in the corresponding Data Use Agreement. The IQVIA PharMetrics Plus Healthcare Claims Database has been evaluated and certified by an independent third party to be in compliance 
with the Health Insurance Portability and Accountability Act (HIPAA) of 1996 statistical de-identification standards and to satisfy the conditions set forth in Sections 164.514 (a)-(b)1ii of the HIPAA Privacy Rule regarding the determination and documentation of statistically de-identified data. Use of data from the IQVIA PharMetrics Plus Healthcare Claims Database for the research described herein was therefore fully compliant with the HIPAA Privacy Rule and federal guidance on Public Welfare and the Protection of Human Subjects (Department of Health and Human Services 2013). Consistent with the use of de-identified data, this study was determined to be exempt from institutional review board approval and informed consent from study participants was not required.

\section{Patient Population}

Adults (aged 18 years or more) with evidence of NMOSD (at least one principal or secondary diagnosis code for NMO [International Classification of Diseases, Ninth Revision: 341.0; International Classification of Diseases, Tenth Revision: G36.0] in the acute care hospital/inpatient setting; or at least two diagnosis codes for NMO at least 30 days apart in the ambulatory/outpatient setting) between January 1, 2013 and March 31, 2018 were included in the analysis. For patients with at least one diagnosis code for NMO in the acute care hospital/inpatient setting, the index date was defined as the date of the first hospitalization. For patients with at least two diagnosis codes for NMO in the ambulatory/outpatient setting, the index date was defined as the date of the second qualifying encounter. Patients were excluded from the NMOSD cohort if they had less than 6 months of health plan enrollment after the index date or if they had evidence of both MS (International Classification of Diseases, Ninth Revision: 340; International Classification of Diseases, Tenth Revision: G35) and MS-related pharmacotherapy (see Appendix A in the Electronic Supplementary Material) after the index date.
A second cohort of "comparison patients" (adults without evidence of NMOSD) were matched 1:1 (without replacement) with patients in the NMOSD cohort. Comparison patients were matched on the basis of age, sex, geographic region, and health plan type. For comparison patients, the index date was defined by the index date of the matched patient with NMOSD. Comparison patients were limited to those with at least 6 months of health plan enrollment after the index date and those with no evidence of both MS and MS-related pharmacotherapy after the index date.

\section{Baseline Characteristics}

Baseline characteristics-including patient demographics, core clinical characteristics, comorbidity profiles, NMOSD-associated conditions, treatment history, and differential diagnoses (see Appendices B-E in the Electronic Supplementary Material)_for patients included in the analysis were ascertained during the 12-month period before the index date. However, patients were not required to have at least 12 months of data before the index date to be included in the study.

\section{Outcomes}

Healthcare utilization, healthcare expenditure, and NMOSD relapses were evaluated during the follow-up period, which began on the index date and ended on the date of health plan disenrollment, the date of admission for hospice care, or the end of the study period (March 31, 2019), whichever occurred first. For patients with evidence of an NMOSD relapse beginning before and ending after the index date, the follow-up period began after the relapse ended. The duration of follow-up thus varied across patients in the study population and spanned a maximum of 6 years.

NMOSD relapses were defined by any of the following criteria: (1) admission to an acute care hospital with a principal diagnosis code for NMO, transverse myelitis, or optic neuritis; (2) admission to an acute care hospital with selected principal diagnosis codes (see Appendix F in 
the Electronic Supplementary Material) and a secondary diagnosis code for NMO, transverse myelitis, or optic neuritis; (3) ambulatory encounters for administration of methylprednisolone (at least two consecutive doses); or (4) ambulatory encounters for plasma exchange or intravenous immunoglobulin administration (see Appendix G in the Electronic Supplementary Material). Qualifying admissions to an acute care hospital and/or qualifying ambulatory encounters occurring within 30 days of each other were considered to be part of the same relapse. Relapse days were quantified from the first to last evidence of a given relapse.

Among patients with NMOSD, healthcare utilization and healthcare expenditure (reported in 2018 US dollars) were evaluated overall (all-cause) and by the amount attributable to NMOSD relapses. These outcomes were analyzed by care setting (hospital/ inpatient, ambulatory/outpatient, or outpatient pharmacy) and by reason for encounter (based on diagnosis or procedure/drug codes). Allcause healthcare utilization and healthcare expenditure were similarly analyzed for comparison patients.

\section{Statistical Analysis}

Baseline characteristics were analyzed using descriptive statistics. Relapse rates, relapse days, healthcare utilization, and healthcare expenditure were summarized using means, frequencies, and corresponding 95\% confidence intervals (CIs); 95\% CIs were estimated using non-parametric bootstrapping. These outcomes were annualized-as needed-to account for variable durations of follow-up and were reported per patient or per relapse. All analyses were conducted using SAS Proprietary Software, Release 9.4 (SAS Institute Inc., Cary, NC, USA).

\section{RESULTS}

\section{Patient Characteristics}

In total, 1363 qualifying patients with NMOSD were identified in the database and included in the study population (Fig. 1). The baseline characteristics of patients with NMOSD are reported in Table 1. The mean age was 44.9 years (standard deviation 13.3 years), and $75.3 \%$ of the patients were female. Core clinical characteristics of the disease before a diagnosis of NMOSD included optic neuritis only (28.2\%), transverse myelitis only (19.1\%), and optic neuritis plus transverse myelitis $(6.1 \%)$. Neuropathic pain $(22.7 \%)$, bladder dysfunction (22.6\%), hemiplegia/paraplegia (19.9\%), and hypothyroidism (13.2\%) were the most common NMOSD-associated conditions, and nearly one-half $(44.3 \%)$ of patients had a prior diagnosis of MS; $29.1 \%$ had previously received methylprednisolone, and $16.5 \%$ had previously received rituximab. Common comorbidities included hypertension (30.0\%), anxiety $(19.5 \%)$, diabetes $(12.8 \%)$, lung disease $(11.7 \%)$, and cerebrovascular disease (10.6\%) (see Table S1 in the Electronic Supplementary Material for the baseline characteristics of comparison patients).

\section{NMOSD Relapses}

Among patients with NMOSD, the median duration of the follow-up period was 2.0 years (interquartile range 2.2 years). During the follow-up, $47.7 \%$ of patients with NMOSD had evidence of one or more relapses (Fig. 2a), resulting in an annualized relapse rate of 0.8 (95\% CI 0.7-0.9), with more relapses requiring ambulatory/outpatient care than hospital/inpatient care (Table 2). The annualized number of relapse days was 12.8 days (95\% CI 11.2-14.4) (Table 2 ), and $17.4 \%$ of patients had relapses lasting at least 20 days (Fig. 2b).

\section{All-Cause Healthcare Utilization and Healthcare Expenditure}

The mean annualized all-cause healthcare expenditure among patients with NMOSD was $\$ 60,599$ (95\% CI \$52,112-66,716) compared with \$8912 (95\% CI \$7084-10,727) among comparison patients, representing a difference of $\quad \$ 51,687 \quad(95 \% \quad$ CI $\quad \$ 43,820-58,664)$ attributable to NMOSD (Table 3). These 


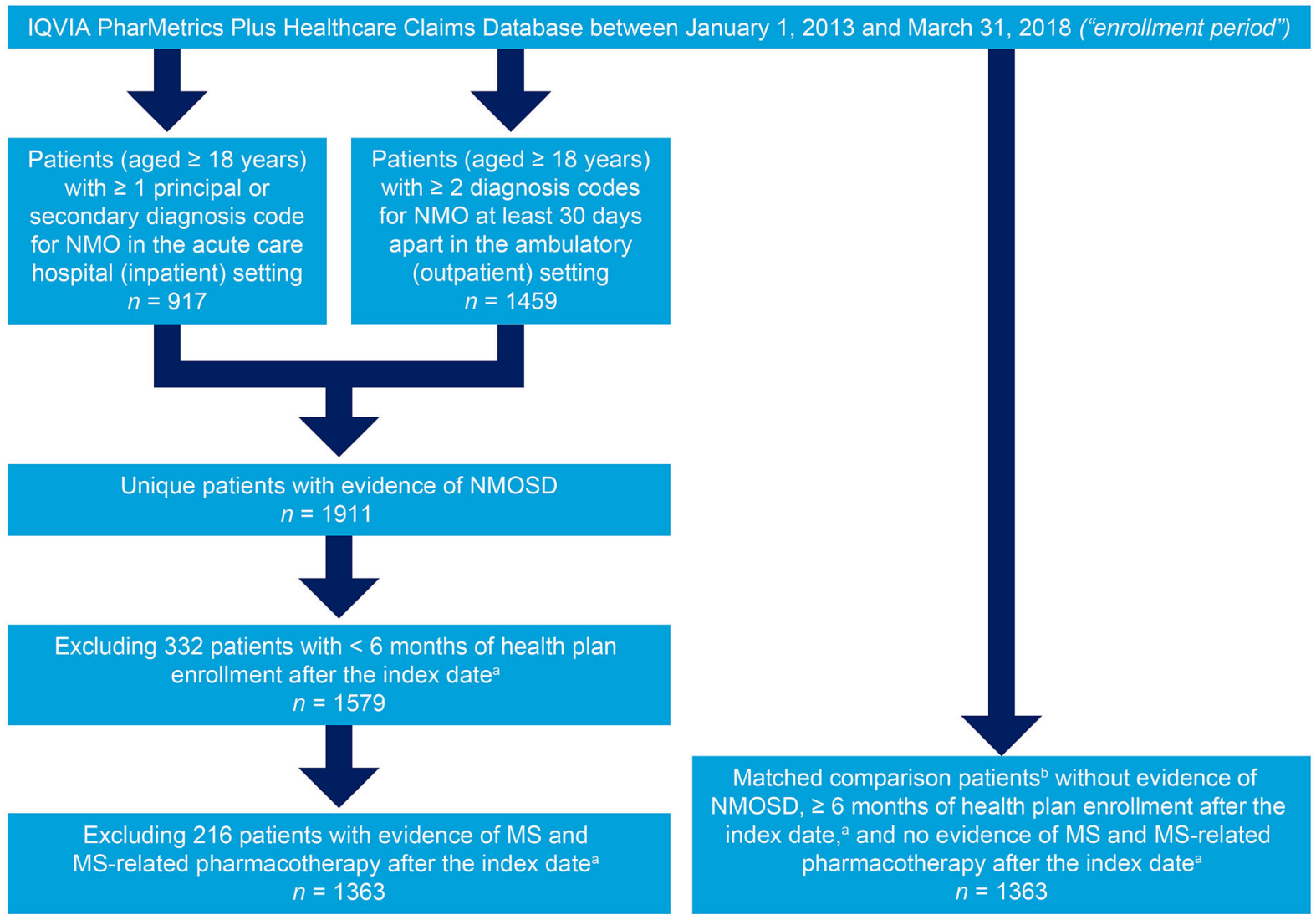

Fig. 1 Identification of patients with NMOSD and matched comparison patients. ${ }^{a}$ For patients with at least one diagnosis code for NMO in the acute care hospital/ inpatient setting, the index date was defined as the date of the first hospitalization. For patients with at least two diagnosis codes for NMO in the ambulatory/outpatient setting, the index date was defined as the date of the second qualifying encounter. For comparison patients, the

increased costs were associated with more hospital/inpatient admissions, longer lengths of hospital stay, and greater numbers of ambulatory/outpatient encounters and outpatient pharmacy prescriptions among patients with NMOSD than among comparison patients (Table 3) (see Tables S2 and S3 in the Electronic Supplementary Material for all-cause healthcare utilization and healthcare expenditure by reason for encounter among patients with NMOSD and matched comparison patients).

index date was defined by the index date of the matched patient with NMOSD. ${ }^{\mathrm{b}}$ Comparison patients were matched on the basis of age, sex, geographic region, and health plan type. MS, multiple sclerosis; NMO, neuromyelitis optica; NMOSD, neuromyelitis optica spectrum disorder

\section{Healthcare Utilization and Healthcare Expenditure Attributed to NMOSD Relapses}

Of all the NMOSD relapses recorded during the follow-up period, $35.0 \%$ were treated by hospital/inpatient admission (Table 4). The mean duration of these relapses was 17.5 days (95\% CI 16.0-19.4), and the corresponding mean expenditure per relapse was $\$ 25,250$ (95\% CI $\$ 23,664-26,957)$. The mean duration of relapses requiring ambulatory/outpatient care only (65.0\% of all NMOSD relapses) was 15.9 days (95\% CI 15.3-15.9), with a corresponding mean 
Table 1 Baseline characteristics of patients with NMOSD

\section{Characteristic $^{\mathrm{a}}$}

Age, years

Mean (SD)

Groups, \%

18-34 years

$35-44$ years

$45-54$ years

$55-64$ years

$\geq 65$ years

Sex, \%

Female

Male

Geographic region, \%

Midwest

South

Northeast

West

Health plan, \%

Health maintenance organization

14.7

Preferred provider organization

75.6

Point of service

5.0

Other/none/unknown/missing

4.6

Core clinical characteristics, $\%$

Optic neuritis only

Transverse myelitis only

19.1

Optic neuritis plus transverse myelitis 6.1

None/no evidence 46.6

NMOSD-associated conditions, \%

Neuropathic pain

Bladder dysfunction

Hemiplegia/paraplegia

Hypothyroidism

Systemic lupus erythematosus

5.1

Sjogren's syndrome

2.0

4.6

$4.9(13.3)$

2

7

4


Table 1 continued

\section{Characteristic $^{\text {a }}$}

Sarcoidosis

Pernicious anemia

Antiphospholipid syndrome

Myasthenia gravis

Ulcerative colitis

Idiopathic thrombocytopenic purpura

Comorbidity profile, $\%$

Hypertension

Anxiety

Diabetes

Lung disease

Cerebrovascular disease

Rheumatic disease

Depression

Skin ulcers/cellulitis

Malignancy

Osteoporosis

Coronary heart disease

Renal disease

Heart failure

Differential diagnoses, \%

Multiple sclerosis

Neuro-Behçet's disease

Parainfectious disorders

Treatment history, \%

Azathioprine

Mycophenolate mofetil

Rituximab

IV methylprednisolone

Plasma exchange
Patients with NMOSD $(n=1363)$

2.3

2.2

1.7

1.6

0.7

0.1

19.5

12.8

11.7

10.6

9.6

8.9

8.5

6.2

5.6

4.4

3.7

2.8

44.3

0.1

2.2

4.8

7.8

16.5

29.1

9.2 
Table 1 continued

\begin{tabular}{ll}
\hline Characteristic $^{\mathbf{a}}$ & Patients with NMOSD $(\boldsymbol{n}=\mathbf{1 3 6 3})$ \\
\hline IV immunoglobulin & 2.6 \\
\hline
\end{tabular}

$I V$ intravenous, NMOSD neuromyelitis optica spectrum disorder, $S D$ standard deviation

a Baseline characteristics were ascertained during the 12-month history period; mean (SD) duration of the history period was 11.3 (1.6) months for patients with NMOSD

(a)

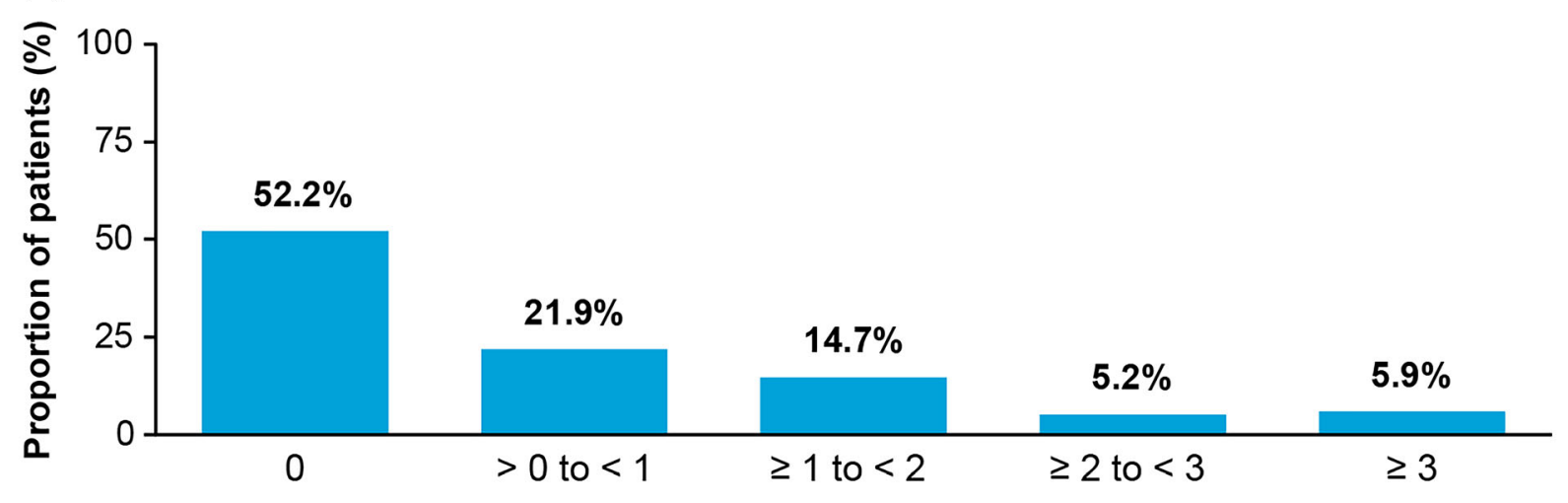

Annualized number of relapses

(b)

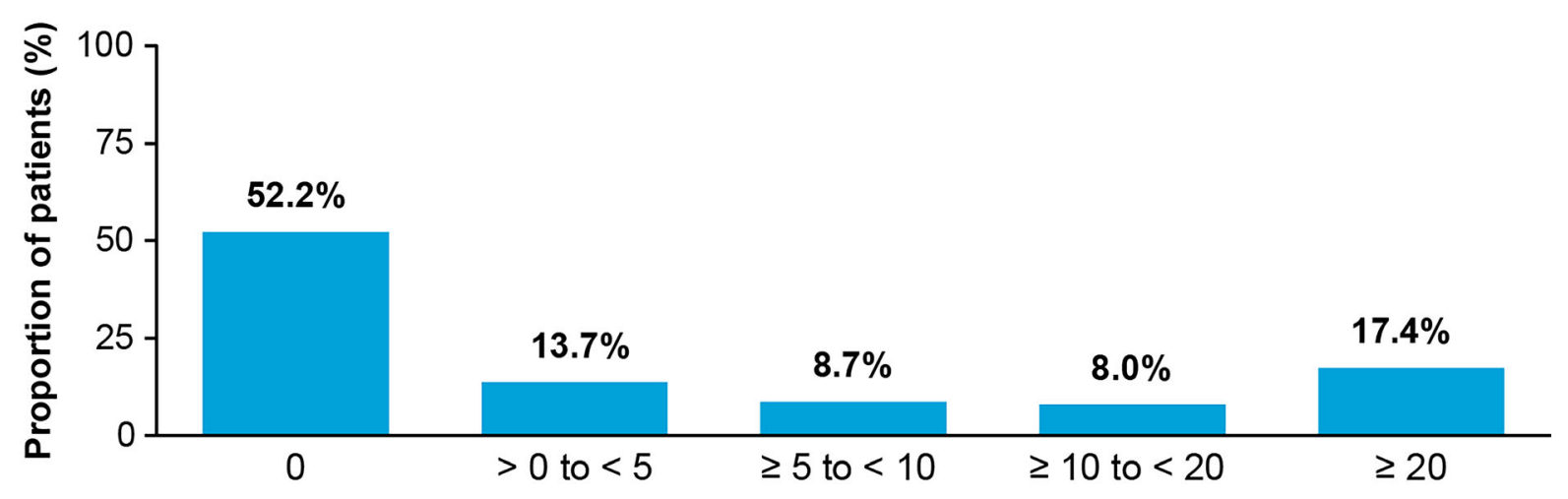

Annualized number of relapse days

Fig. 2 Distribution of patients with NMOSD stratified by annualized number of $\mathbf{a}$ relapses and $\mathbf{b}$ relapse days. NMOSD, neuromyelitis optica spectrum disorder

expenditure of $\$ 4550$ (95\% CI \$4344-4691) per relapse (Table 4).

When analyzing healthcare expenditure per patient, the mean annualized total expenditure for NMOSD relapses was \$10,070 (95\% CI $\$ 7726-12,660)$, with hospital/inpatient care requiring more expenditure than ambulatory/ outpatient care (Table 2). The mean annualized healthcare expenditure increased on the basis of the total number of relapses, with $5.9 \%$ of patients who had three or more annualized 
Table 2 Annualized rate of relapses, relapse days, and healthcare expenditure for relapses (per patient) in patients with NMOSD

Patients with NMOSD $(n=1363)$

Annualized rate of relapses and relapse days, mean (95\% CI)

Annualized number of relapses

$0.8(0.7-0.9)$

Annualized number of relapses requiring hospital/inpatient care

$0.3(0.3-0.4)$

Annualized number of relapses requiring ambulatory/outpatient care only

$0.5(0.4-0.5)$

Annualized number of relapse days

$12.8(11.2-14.4)$

Annualized healthcare expenditure ${ }^{\mathrm{a}}$ for relapses, mean (95\% CI)

Hospital/inpatient care

$\$ 8130(\$ 6060-10,835)$

Ambulatory/outpatient care only

\$1940 (\$1254-2819)

Total

$\$ 10,070(\$ 7726-12,660)$

CI confidence interval, NMOSD neuromyelitis optica spectrum disorder

${ }^{a}$ Reported in 2018 US dollars

relapses accruing mean annualized healthcare expenditures of over $\$ 83,000$ (Fig. 3b).

\section{DISCUSSION}

Relapses in NMOSD are unpredictable and can be devastating, leading to permanent disability. Our findings suggest that relapses are common in patients with NMOSD and that a subset of severely affected patients (those with three or more relapses) account for a disproportionate burden of disease. Our findings also indicate that annual healthcare expenditure attributed to the treatment and management of NMOSD is considerable (more than $\$ 50,000$ per patient in total) in both the hospital/inpatient and ambulatory/outpatient settings, and, in particular, among the subset of patients who experienced relapses. In addition, our findings show that relapses are costly, averaging $\$ 11,883$ per relapse overall and $\$ 25,250$ per relapse requiring hospitalization (35.0\% of all relapses).

Although, to the best of our knowledge, our study provides the first published evidence on the economic burden of NMOSD relapses and the management of patients with NMOSD in a peer-reviewed journal, our findings are largely comparable with those presented at recent medical conferences, notwithstanding differences in study designs and study populations $[13,14]$. Compared with recent publications, the baseline characteristics of our NMOSD cohort (mean age 44.9 years; $75.3 \%$ of patients were female) largely matched the profile of patients reported in other notable studies (e.g., the German Neuromyelitis Optica Study [NEMOS] cohort was reported to have a mean age of 41 years and $83 \%$ of the population was female [15]; and data from the French network on MS and NMOSD [OFSEP and NOMADMUS] study groups showed similar results [16]). In addition, the annualized relapse rate in our study was 0.8 , while the annualized relapse rate in the NEMOS study was 0.6 [15] and ranged from 0.2 in treated patients to 0.9 in untreated patients in the OFSEP/NOMADMUS cohort [16].

Regarding economic outcomes, Exuzides et al. evaluated all-cause healthcare expenditure among patients with NMOSD and matched comparison patients using data from a US commercial claims database from 2014 to 2018 [13]. The mean total all-cause healthcare expenditure (reported in 2019 US dollars) was $\$ 62,682$ among patients with NMOSD $(n=162$; mean age 43 years; $74 \%$ were female), versus $\$ 8270$ among comparison patients $(n=810)$; the NMOSD-attributable expenditure thus 
Table 3 Annualized all-cause healthcare utilization and expenditure among patients with NMOSD and matched comparison patients

$$
\begin{array}{ll}
\begin{array}{l}
\text { Patients with NMOSD } \\
(n=1363)
\end{array} & \begin{array}{l}
\text { Comparison patients } \\
(n=1363)
\end{array} \\
\text { Difference }^{\mathrm{b}}
\end{array}
$$

Annualized all-cause healthcare utilization

Hospital/inpatient care

Acute care facilities

Patients with $\geq 1$ hospitalization,

$49.3(46.1-51.2)$

$10.3(8.9-11.8)$

$39.0(34.6-41.2)$

$\%(95 \% \mathrm{CI})$

Number of hospitalizations, mean

$0.9(0.8-1.0)$

$0.1(0.1-0.1)$

$0.8(0.6-0.9)$

(95\% CI)

Number of hospital days, mean

$11.3(9.0-13.4)$

$0.1(0.1-0.2)$

$11.2(8.9-13.3)$

(95\% CI)

Number of ICU days, mean (95\%

$1.4(1.0-1.9)$

$0.0(0.0-0.0)$

$1.4(1.0-1.9)$

CI)

Rehabilitation facilities

Number of admissions, mean

$0.2(0.1-0.4)$

$0.0(0.0-0.0)$

$0.2(0.1-0.4)$

(95\% CI)

Number of rehabilitation days,

$0.3(0.1-0.7)$

$0.0(0.0-0.0)$

$0.3(0.1-0.7)$

mean $(95 \% \mathrm{CI})$

Other facilities

Number of admissions, mean

(95\% CI)

Number of inpatient days, mean (95\% CI)

$0.8(0.4-1.1)$

$0.0(0.0-0.1)$

$0.8(0.4-1.1)$

$2.1(1.2-3.0)$

$0.0(0.0-0.0)$

$2.1(1.2-3.0)$

Ambulatory/outpatient care

Patients with $\geq 1$ encounter, \% (95\% CI)

All care settings

$99.9(99.6-100.0)$

$90.1(88.3-91.3)$

$9.8(8.1-11.3)$

Emergency department

$44.5(41.8-46.6)$

$20.0(17.2-22.4)$

24.6 (20.4-27.5)

Physician office

98.3 (97.6-98.9)

$88.1(86.4-89.6)$

$10.2(8.1-11.5)$

Hospital outpatient

$92.2(90.9-93.5)$

$60.0(57.5-62.3)$

$32.2(29.0-35.0)$

Other

$79.0(77.0-81.1)$

$58.2(55.1-60.3)$

$20.8(17.9-24.5)$

Number of encounters, mean (95\% CI)

All care settings

$32.0(30.2-33.7)$

$14.7(13.1-15.7)$

$17.3(15.1-19.4)$

Emergency department

$0.5(0.4-0.6)$

$0.2(0.1-0.3)$

$0.3(0.2-0.4)$

Physician office

$15.4(14.5-15.9)$

$11.3(10.3-12.4)$

$4.1(2.8-4.9)$

Hospital outpatient

$7.2(6.6-7.7)$

$2.0(1.7-2.2)$

$5.2(4.5-5.9)$ 
Table 3 continued

\begin{tabular}{lccc}
\hline & $\begin{array}{l}\text { Patients with NMOSD } \\
(\boldsymbol{n}=\mathbf{1 3 6 3})\end{array}$ & $\begin{array}{l}\text { Comparison patients }^{\mathbf{a}} \\
(\boldsymbol{n}=\mathbf{1 3 6 3})\end{array}$ & Difference $^{\mathbf{b}}$ \\
\hline Other & $8.9(7.0-10.0)$ & $1.3(1.1-1.4)$ & $7.6(5.8-8.8)$ \\
Outpatient pharmacy & & & \\
Number of prescriptions & $33.4(30.9-35.0)$ & $21.0(18.3-23.3)$ & $12.4(8.8-15.0)$ \\
dispensed, mean $(95 \% \mathrm{CI})$ & & & \\
All-cause healthcare expenditures, ${ }^{\mathbf{c}}$ mean & $(95 \% \mathrm{CI})$ & & \\
Hospital/inpatient care & $\$ 27,617(\$ 21,724-33,954)$ & $\$ 2150(\$ 1241-3111)$ & $\$ 25,466(\$ 19,967-32,235)$ \\
Acute care facilities & $\$ 26,568(\$ 20,946-32,541)$ & $\$ 2121(\$ 1403-2836)$ & $\$ 24,447(\$ 18,285-30,399)$ \\
Rehabilitation facilities & $\$ 182(\$ 26-415)$ & $\$ 0(\$ 0-1)$ & $\$ 181(\$ 26-414)$ \\
Other facilities & $\$ 867(\$ 215-1899)$ & $\$ 29(\$ 6-65)$ & $\$ 838(\$ 165-1872)$ \\
Ambulatory/outpatient care & $\$ 29,282(\$ 26,715-31,592)$ & $\$ 4788(\$ 3743-5895)$ & $\$ 24,494(\$ 22,364-26,731)$ \\
Emergency department & $\$ 670(\$ 539-804)$ & $\$ 259(\$ 171-362)$ & $\$ 411(\$ 251-567)$ \\
Physician office & $\$ 6303(\$ 5455-6942)$ & $\$ 1568(\$ 1223-1873)$ & $\$ 4735(\$ 3886-5452)$ \\
Hospital outpatient & $\$ 17,328(\$ 15,354-19,325)$ & $\$ 2230(\$ 1728-2794)$ & $\$ 15,097(\$ 12,614-17,118)$ \\
Other & $\$ 4982(\$ 3933-6105)$ & $\$ 731(\$ 487-1033)$ & $\$ 4251(\$ 3198-5194)$ \\
Outpatient pharmacy & $\$ 3700(\$ 3208-4284)$ & $\$ 1973(\$ 1476-2435)$ & $\$ 1726(\$ 973-2406)$ \\
Total & $\$ 60,599(\$ 52,112-66,716)$ & $\$ 8912(\$ 7084-10,727)$ & $\$ 51,687(\$ 43,820-58,664)$ \\
\hline
\end{tabular}

$C I$ confidence interval, $I C U$ intensive care unit, NMOSD neuromyelitis spectrum disorder, $S D$ standard deviation

a The median (interquartile range) duration of the follow-up period was 2.0 (2.2) years for patients with NMOSD and 2.1 (2.3) years for comparison patients

b The difference was calculated as the NMOSD-attributable utilization and expenditure minus the utilization and expenditure of comparison patients

${ }^{c}$ Reported in 2018 US dollars

totaled $\$ 54,412$. Stafkey-Mailey et al. evaluated relapse frequency and annual healthcare costs among patients with NMOSD using data from a large US healthcare claims database from 2009 to 2014 [14]. Among the 1349 patients (mean age 46 years; $68 \%$ were female) in their study, $23 \%$ had one or more relapses during the 12-month follow-up period, and the mean number of relapses among patients with one or more relapses was 1.8 . The mean total all-cause healthcare expenditure (reported in 2014 US dollars) during the follow-up period was $\$ 40,080$ among all patients with NMOSD,
$\$ 78,034$ among those with one or more relapse, and $\$ 28,803$ among those without a relapse.

Our estimates, along with recently presented research, suggest that the economic burden of NMOSD is comparable with, and may be higher than, the economic burden of other CNS disorders. In a study of 15,902 commercially insured patients with MS in the USA, mean annual healthcare charges (reported in 2011 US dollars) were estimated to be $\$ 27,000$, and increased with worsening disability (mildmoderate disability, $\$ 30,000$; moderate disability, $\$ 50,000$; severe disability, more than $\$ 100,000)$ [17]. In a more recent evaluation of 
Table 4 Healthcare utilization and expenditure during NMOSD relapses

Patients with NMOSD $(n=1363)$

Relapses requiring hospital/inpatient care

Percentage of all relapses (95\% CI)

$35.0(34.0-37.0)$

Healthcare utilization (per relapse)

Duration (days), mean (95\% CI)

$17.5(16.0-19.4)$

Length of hospital stay (days), mean (95\% CI)

$14.6(13.9-15.2)$

Numbers of encounters, mean ( $95 \% \mathrm{CI})$

Hospitalizations

$1.6(1.6-1.7)$

Ambulatory encounters

$0.4(0.3-0.4)$

Principal diagnosis, \% (95\% CI)

$\mathrm{NMO}$

$66.0(66.0-68.0)$

Optic neuritis

$10.0(8.0-11.0)$

Transverse myelitis

$20.0(18.0-21.0)$

Other

$47.0(47.0-49.0)$

Healthcare expenditure ${ }^{a}$ (per relapse), mean (95\% CI)

Hospital/inpatient care

$\$ 24,917(\$ 23,767-26,560)$

Ambulatory/outpatient care

$\$ 333(\$ 264-430)$

Total

Relapses requiring ambulatory/outpatient care only

Percentage of all relapses (95\% CI)

$65.0(62.0-67.0)$

Healthcare utilization (per relapse)

Duration (days), mean (95\% CI)

$15.9(15.3-15.9)$

Number of ambulatory/outpatient encounters, mean (95\% CI)

$2.4(2.4-2.5)$

Care setting, \% (95\% CI)

Emergency department

$2.0(1.0-2.0)$

Physician office

$32.0(31.0-35.0)$

Hospital outpatient

$55.0(52.0-56.0)$

Other

$21.0(19.0-22.0)$

Diagnoses, \% (95\% CI)

$\mathrm{NMO}$

$53.0(51.0-54.0)$

Optic neuritis

$6.0(5.0-7.0)$

Transverse myelitis

$2.0(2.0-3.0)$

Procedures/drugs, \% (95\% CI)

IV methylprednisolone

$59.0(58.0-61.0)$ 
Table 4 continued

\begin{tabular}{lc}
\hline & Patients with NMOSD $(\boldsymbol{n}=\mathbf{1 3 6 3})$ \\
\hline IV immunoglobulin & $38.0(36.0-41.0)$ \\
Plasma exchange & $11.0(10.0-12.0)$ \\
Healthcare expenditure $^{a}$ (per relapse), mean (95\% CI) & $\$ 4550(\$ 4344-4691)$ \\
Relapses requiring hospital/inpatient or ambulatory/outpatient care only & \\
Healthcare utilization (per relapse) & $16.5(16.1-17.2)$ \\
Duration (days), mean (95\% CI) & $0.6(0.5-0.6)$ \\
Numbers of encounters, mean (95\% CI) & $1.7(1.6-1.7)$ \\
Hospitalizations & \\
Ambulatory encounters & \\
Healthcare expenditure ${ }^{a}$ (per relapse), mean (95\% CI) & $\$ 8827(\$ 7979-10,215)$ \\
Hospital/inpatient care & $\$ 3056(\$ 2662-3437)$ \\
Ambulatory/outpatient care & $\$ 11,883(\$ 10,663-13,613)$ \\
Total &
\end{tabular}

$C I$ confidence interval, $I V$ intravenous, $N M O$ neuromyelitis optica, $N M O S D$ neuromyelitis spectrum disorder

${ }^{\text {a }}$ Reported in 2018 US dollars

patients with MS, the annual total healthcare expenditure (reported in 2015 US dollars) was estimated to be $\$ 62,500$ per patient [18].

\section{Limitations}

While healthcare claims databases provide information on large numbers of patients with specific diagnoses, several limitations of the use of such databases should be noted. First, although similar algorithms for identifying patients with NMOSD have been used in prior studies [12-14], to the best of our knowledge, these algorithms have not been formally evaluated against a "gold standard" and thus their accuracy (i.e., sensitivity and specificity) is unknown. Similarly, the use of operational algorithms to identify NMOSD relapses and characterize patient comorbidity profiles undoubtedly resulted in some misclassification (i.e., false positives and false negatives). In addition, although the presence of an AQP4 antibody test can be ascertained from claims data, results of the test are not available. Thus, AQP4 serostatus cannot be determined for patients in the NMOSD cohort. Furthermore, as with all retrospective database analyses, the potential for residual confounding in the data and results cannot be ruled out.

Although the IQVIA PharMetrics Plus Healthcare Claims Database was sufficiently large to robustly evaluate study objectives among all patients with NMOSD in the study population, evaluations of study outcomes within subgroups defined therein may lack adequate precision and should be interpreted with caution. In addition, the true cost of NMOSD-related encounters is not available in the IQVIA PharMetrics Plus Healthcare Claims Database, and thus expenditures (i.e., amounts paid by health plans for services rendered) were employed. Because the study database includes only commercially insured patients, who may have less severe disease than other patients (e.g., 
(a)

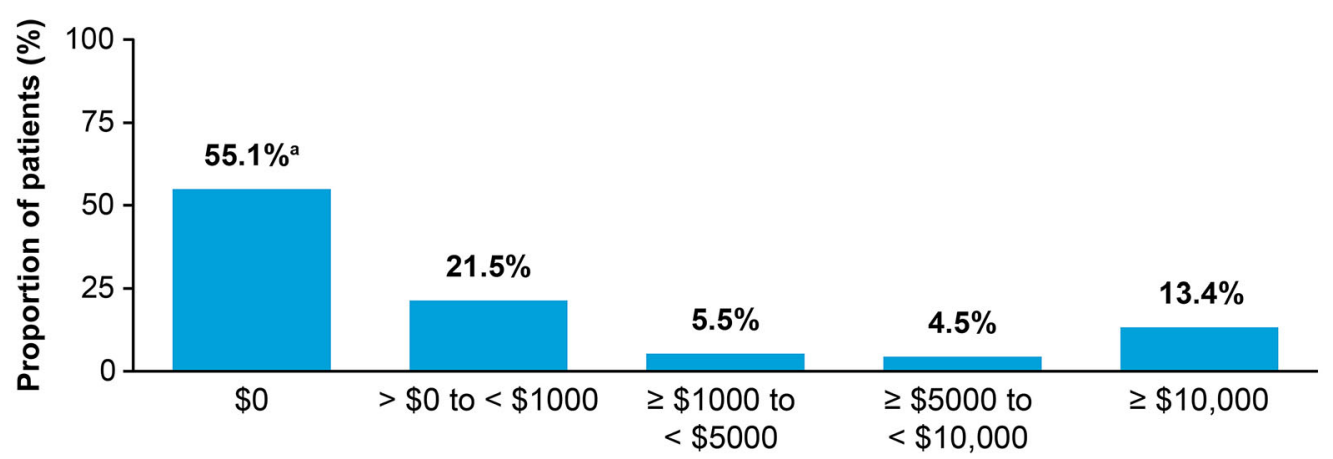

Annualized healthcare expenditure

(b)

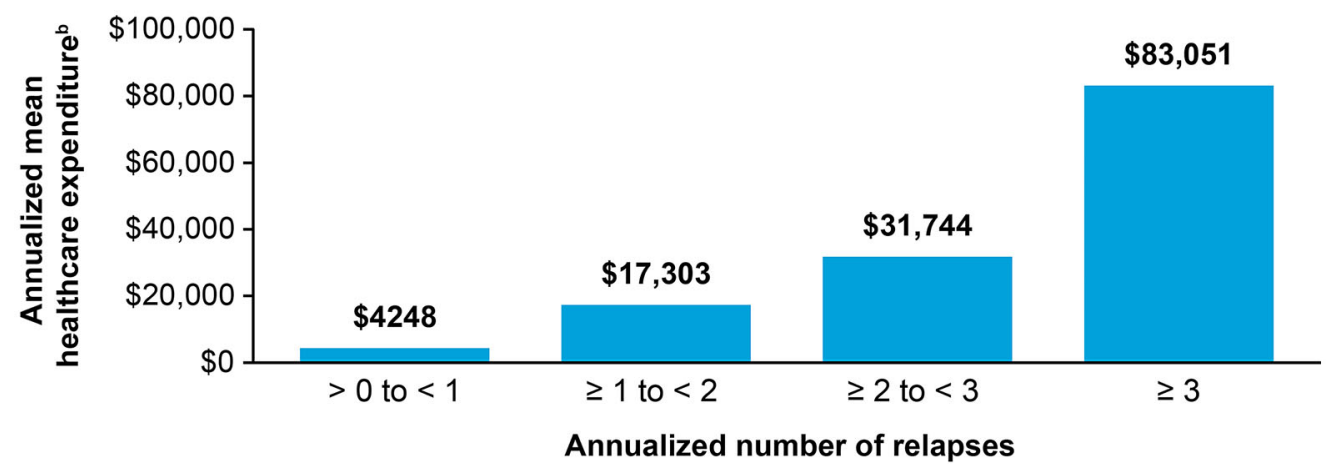

Fig. 3 Distribution of patients with NMOSD by annualized healthcare expenditure. a Proportion of patients stratified by annualized healthcare expenditure. b Annualized mean healthcare expenditure by annualized number of

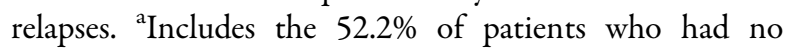

Medicare beneficiaries who are blind or have other disabilities), the reported burden of NMOSD may have been underestimated. Also, the possibility exists that patients may have left a participating health plan (i.e., a health plan that contributes data to the IQVIA PharMetrics Plus Healthcare Claims Database) to join another contributing health plan; thus, these patients could be represented multiple times in the study database during different periods. The extent of this phenomenon is believed to be low and, given the objectives of this research, its impact is believed to be analytically minor. Finally, adults with public health insurance (e.g., Medicare fee-for-service) and adults without health insurance are not represented in the study database; therefore, caution should be evidence of a relapse, plus 40 patients who had evidence of relapses with no associated costs. ${ }^{\text {b }}$ Reported in 2018 US dollars. NMOSD, neuromyelitis optica spectrum disorder

used when generalizing the study results to other populations and settings.

\section{CONCLUSIONS}

The findings of this large, retrospective, observational study of US commercial claims data indicate that relapses among patients with NMOSD are common in clinical practice. These relapses are associated with substantial healthcare utilization and expenditure. Therapies with the highest relapse risk reduction could lead to markedly lower relapse-associated healthcare utilization and clinical burden in patients with NMOSD. 


\section{ACKNOWLEDGEMENTS}

Funding. Sponsorship for all aspects of this research, including data analysis, medical writing, and editorial assistance, was provided by Alexion Pharmaceuticals Inc., Boston, MA, USA. Funding for the journal's Rapid Service Fee was also provided by Alexion Pharmaceuticals Inc., Boston, MA, USA.

Medical Writing and Editorial Assistance. Medical writing and editorial support were provided by Steven F. Merkel, PhD, of Oxford PharmaGenesis, Inc., Newtown, PA, USA. Sponsorship for this assistance was provided by Alexion Pharmaceuticals Inc., Boston, MA, USA.

Authorship. All named authors meet the International Committee of Medical Journal Editors (ICMJE) criteria for authorship for this article, take responsibility for the integrity of the work as a whole, and have given their approval for this version to be published.

Authors' Contributions. The contribution of each of these persons to this study is as follows: (1) conception and design (all authors), acquisition of data (Kielhorn, Weycker), analysis or interpretation of data (all authors); and (2) preparation of manuscript (Houde, Shaff, Weycker), critical review of manuscript (Royston, Kielhorn, Tanvir, Bhattacharyya, Levy). The study sponsor reviewed the study research plan and study manuscript; data management, processing, and analyses were conducted by Policy Analysis Inc. (PAI), and all final analytic decisions were made by study investigators. All authors have read and approved the final version of the manuscript.

Prior Presentation. This manuscript is based on work previously presented at MS Virtual 2020, the 8th Joint ACTRIMS-ECTRIMS Meeting, which was held virtually on September 11-13, 2020.

Disclosures. Minying Royston, Adrian Kielhorn, and Imran Tanvir are employed by, and own shares issued by, Alexion Pharmaceuticals Inc. Derek Weycker, Melody Shaff, and Linnea Houde are employed by Policy Analysis Inc. (PAI), which received funding for this research from Alexion Pharmaceuticals Inc. Shamik Bhattacharyya has consulted for Alexion Pharmaceuticals Inc., and Teladoc Health Inc., and has received publishing honoraria from Springer and UpToDate. Michael Levy has received consulting fees from all three manufacturers involved in NMOSD clinical trials: Alexion Pharmaceuticals Inc., Genentech/ Roche/Chugai, and Viela Bio. In addition, he has received consulting fees from Mitsubishi, Quest Diagnostics, and UCB Pharmaceuticals.

Compliance with Ethics Guidelines. The study database was de-identified before its release to study investigators, as set forth in the corresponding Data Use Agreement. The IQVIA PharMetrics Plus Healthcare Claims Database has been evaluated and certified by an independent third party to be in compliance with the Health Insurance Portability and Accountability Act (HIPAA) of 1996 statistical de-identification standards and to satisfy the conditions set forth in Sections 164.514 (a)-(b)1ii of the HIPAA Privacy Rule regarding the determination and documentation of statistically deidentified data. Use of data from the IQVIA PharMetrics Plus Healthcare Claims Database for the research described herein was therefore fully compliant with the HIPAA Privacy Rule and federal guidance on Public Welfare and the Protection of Human Subjects (Department of Health and Human Services 2013). Consistent with the use of de-identified data, this study was determined to be exempt from institutional review board approval and informed consent from study participants was not required.

Data Availability. The data are proprietary, provided by a third-party vendor, and the authors do not have permission to disseminate the data without approval of the vendor.

Open Access. This article is licensed under a Creative Commons Attribution-NonCommercial 4.0 International License, which permits any non-commercial use, sharing, adaptation, 
distribution and reproduction in any medium or format, as long as you give appropriate credit to the original author(s) and the source, provide a link to the Creative Commons licence, and indicate if changes were made. The images or other third party material in this article are included in the article's Creative Commons licence, unless indicated otherwise in a credit line to the material. If material is not included in the article's Creative Commons licence and your intended use is not permitted by statutory regulation or exceeds the permitted use, you will need to obtain permission directly from the copyright holder. To view a copy of this licence, visit http://creativecommons.org/licenses/by$\mathrm{nc} / 4.0 /$.

\section{REFERENCES}

1. Borisow N, Mori M, Kuwabara S, Scheel M, Paul F. Diagnosis and treatment of NMO spectrum disorder and MOG-encephalomyelitis. Front Neurol. 2018;9: 888.

2. Wingerchuk DM, Banwell B, Bennett JL, et al. International consensus diagnostic criteria for neuromyelitis optica spectrum disorders. Neurology. 2015;85(2):177-89.

3. Abboud H, Petrak A, Mealy M, Sasidharan S, Siddique $\mathrm{L}$, Levy $\mathrm{M}$. Treatment of acute relapses in neuromyelitis optica: steroids alone versus steroids plus plasma exchange. Mult Scler. 2016;22(2): 185-92.

4. Kessler RA, Mealy MA, Levy M. Treatment of neuromyelitis optica spectrum disorder: acute, preventive, and symptomatic. Curr Treat Opt Neurol. $2016 ; 18(1): 2$.

5. Kleiter I, Gahlen A, Borisow N, et al. Neuromyelitis optica: evaluation of 871 attacks and 1,153 treatment courses. Ann Neurol. 2016;79(2):206-16.

6. Kowarik MC, Soltys J, Bennett JL. The treatment of neuromyelitis optica. J Neuroophthalmol. 2014;34(1):70-82.

7. Alexion Pharmaceuticals. Soliris (eculizumab) [package insert]. U.S. Food and Drug Administration website. 2007. https://www.accessdata.fda. gov/drugsatfda_docs/label/2007/125166lbl.pdf. 2007. Accessed 24 Nov 2020.
8. Viela Bio. Uplizna (inebilizumab-cdon) [package insert]. U.S. Food and Drug Administration website. 2020. https://www.accessdata.fda.gov/drugsatfda docs/label/2020/761142s000lbl.pdf. Accessed 24 Nov 2020.

9. Genentech. Enspryng (satralizumab-mwge) [package insert]. U.S. Food and Drug Administration website. 2020. https://www.accessdata.fda.gov/ drugsatfda_docs/label/2020/761149s000lbl.pdf. Accessed 24 Nov 2020.

10. Huda S, Whittam D, Bhojak M, Chamberlain J, Noonan C, Jacob A. Neuromyelitis optica spectrum disorders. Clin Med (Lond). 2019;19(2):169-76.

11. Asgari N, Lillevang ST, Skejoe HPB, Kyvik KO. Epidemiology of neuromyelitis optica spectrum disorder in Denmark (1998-2008, 2007-2014). Brain Behav. 2019;9(7):e01338.

12. Ajmera MR, Boscoe A, Mauskopf J, Candrilli SD, Levy M. Evaluation of comorbidities and health care resource use among patients with highly active neuromyelitis optica. J Neurol Sci. 2018;384: 96-103.

13. Exuzides A, Sheinson D, Sidiropoulos P, et al. Estimating the cost of illness for patients with neuromyelitis optica spectrum disorder from US commercial claims. Poster presented at: MSVirtual2020; 2020 Sept 11. Poster P0712.

14. Stafkey-Mailey D, Boscoe A, Ajmera M, Mauskopf J, Iloeje U, Levy M. A real-world analysis of relapses and costs of neuromyelitis optica spectrum disorders using a United States administrative claims database (P6.161). Neurology. 2016;86(16 Supplement):P6.161.

15. Stellmann JP, Krumbholz M, Friede T, et al. Immunotherapies in neuromyelitis optica spectrum disorder: efficacy and predictors of response. J Neurol Neurosurg Psychiatry. 2017;88(8):639-47.

16. Poupart J, Giovannelli J, Deschamps R, et al. Evaluation of efficacy and tolerability of first-line therapies in NMOSD. Neurology. 2020;94(15): e1645-56.

17. Carroll CA, Fairman KA, Lage MJ. Updated cost-ofcare estimates for commercially insured patients with multiple sclerosis: retrospective observational analysis of medical and pharmacy claims data. BMC Health Serv Res. 2014;14:286.

18. Kim Y, Krause TM, Blum P, Freeman L. Disease modifying therapies continue to drive up health care cost among individuals with multiple sclerosis. Mult Scler Relat Disord. 2019;30:69-75. 